Gynecologic and

Obstetric Investigation
Gynecol Obstet Invest 2014;77:6-13

DOI: $10.1159 / 000355100$
Received: December 20, 2012

Accepted: August 19, 2013

Published online: December 11, 2013

\title{
Maternal Lipid Change in Relation to Length of Gestation: A Prospective Cohort Study with Preconception Enrollment of Women
}

\author{
S. Katherine Laughon ${ }^{a} \quad$ Alexander C. McLain ${ }^{b}$ Rajeshwari Sundaram ${ }^{b}$ \\ Janet M. Catov ${ }^{d, e}$ Germaine M. Buck Louisc \\ ${ }^{a}$ Epidemiology Branch and ${ }^{b}$ Biostatistics Branch, ${ }^{c}$ Division of Intramural Population Health Research, Eunice Kennedy \\ Shriver National Institute of Child Health and Human Development, National Institutes of Health, Rockville, Md., and \\ Departments of ${ }^{\mathrm{d} O b s t e t r i c s, ~ G y n e c o l o g y}$ and Reproductive Services and e Epidemiology, University of Pittsburgh, \\ Pittsburgh, Pa., USA
}

\section{Key Words}

Cholesterol · Lipids · Miscarriage $\cdot$ Preterm birth .

Triglycerides

\begin{abstract}
Background/Aims: We sought to investigate the association between preconception serum lipids and their daily rate of change in relation to length of gestation. Methods: In a cohort of 70 women, 61 (87\%) became pregnant, resulting in 48 (69\%) live births. Serum lipid measurements (in milligrams per deciliter) included total cholesterol, free cholesterol, triglycerides and phospholipids at preconception, upon human chorionic gonadotropin-confirmed pregnancy and following pregnancy loss $(<14$ weeks) or post partum. Pregnancy outcome (loss, preterm and term delivery) and gestational length were modeled relative to daily rate of change in lipids using multinomial regression and Cox proportional hazards models, respectively, adjusting for body mass index and smoking. Results: A rate of triglyceride change below the median was associated with an increased risk for pregnancy loss compared with term birth (adjusted odds ratio: $9.02 ; 95 \% \mathrm{Cl}: 1.62-50.30$ ). A rate of triglyceride change of $\leq 0.01 \mathrm{mg} / \mathrm{dl}$ per day versus above the median was
\end{abstract}

\section{KARGER}

E-Mail karger@karger.com www.karger.com/goi associated with a trend for increased risk of pregnancy loss or preterm ( $<37$ weeks) birth (adjusted hazard ratio: 1.77; 95\% Cl: 0.94-3.33). Conclusion: A low rate of triglyceride change during early pregnancy may be a signal of risk of pregnancy loss or preterm birth. Lipids offer promise for identifying pregnancies at risk for adverse outcomes.

(c) 2013 S. Karger AG, Basel

\section{Introduction}

During pregnancy, maternal lipids are important both for steroidogenesis for the mother, placenta and fetus, as well as for fetal growth [1]. A U-shaped distribution has been reported for prepregnancy serum lipids and shorter gestational length, although most evidence stems from cross-sectional research [2]. The reasons for an association between increased lipids and preterm birth are unclear but may reflect an individual's predisposition to develop features of metabolic syndrome, including elevated triglycerides, lower high-density lipoprotein cholesterol and hyperglycemia [3]. Metabolic syndrome is a disorder marked by increased inflammation, a reported pathway for the preterm birth [4]. Dyslipidemia also has been sug- 
gested to be one pathway that explains why women at risk for pregnancy complications such as preterm birth are also at risk for developing cardiovascular disease later in life [5-7]. While intriguing, this finding does not explain the association between lower lipids and preterm birth. Perhaps without sufficient lipid substrates, placentation is affected, leading to adverse pregnancy outcomes. Thus, low lipid levels may also be a marker for poor maternal health.

The rate of lipid change may also be important for pregnancy outcomes, though the available literature relies upon measurements across pregnancy without taking preconception concentration into account. In one study, a slower rise in triglyceride concentrations in the first half of pregnancy was associated with an increased risk for preterm ( $<34$ weeks) birth [8]. Assessing the rate of change in serum lipids across pregnancy may in part be a function of preconception serum lipids allowing for differing pathways, such that a slower rise in lipids may be indicative of a poor adaptation to pregnancy (i.e. abnormal placentation), placing a woman at risk for adverse pregnancy outcomes such as preterm birth. Similar to the association of elevated preconception lipids with preterm birth, elevated cholesterol or triglycerides early in pregnancy have also been found to be associated with a 2- to 3 -fold increased risk of spontaneous preterm birth $[8,9]$. These associations may simply reflect baseline lipid concentrations prior to pregnancy [10], but critical data gaps remain, since, to our knowledge, no previous studies have examined the trajectory of lipids from preconception through pregnancy to delivery in relation to gestational length. Such information may be important for assessing prenatal risk, and it served as the impetus of study. Using data from a prospective pregnancy cohort with preconception enrollment, our aim was to investigate whether an impaired lipid response from preconception to early gestation would be associated with pregnancy loss or preterm delivery.

\section{Subjects and Methods}

\section{Study Design and Population}

We performed an analysis utilizing a subset of women from the NYSACS (New York State Angler Cohort Study) to assess serum lipids and gestation [11]. The NYSACS randomly recruited licensed anglers, aged 18-40 years from 16 counties along Lakes Erie and Ontario. There were 2,637 women in the study who had indicated possible interest in becoming pregnant in 1995-1996. In 1996-1997, letters were mailed to these women and 1,031 of them could be reached for screening by phone after multiple attempts. There were 244 women with no history of physician-diagnosed infertility and who were planning a pregnancy in the next 6 months. Of these eligible women, 113 were recruited upon discontinuing contraception and followed daily for up to 12 menstrual cycles at risk for pregnancy for a study investigating serum polychlorinated biphenyl concentrations across critical windows of human development [12]. Forty-three women were excluded from the present analysis for various reasons: 14 women were already pregnant, 20 women had incomplete data for establishing pregnancy and gestation history because they withdrew from the study, and 9 women had insufficient serum for lipid quantification. The final cohort comprised 70 women.

\section{Data Collection}

The women underwent an interview prior to conception and completed a daily diary on menstruation, intercourse and pregnancy test results to permit estimation of the timing of conception. They utilized home pregnancy tests on the day of expected menstruation and approximately 1 week later, according to the manufacturer's guidance for determining pregnancy status. Gestational age was thus based on the last menstrual period with human chorionic gonadotropin confirmation. Nonfasting blood specimens were collected before conception, at human chorionic gonadotropin-confirmed pregnancy (pregnancy), after a pregnancy loss or 12 months later in case of no pregnancy. At each time, serum was analyzed for total cholesterol, free cholesterol, triglycerides and phospholipids. All blood samples were kept on ice and transported to the laboratory within $24 \mathrm{~h}$. Total cholesterol, free cholesterol, triglycerides and phospholipids were quantified using enzymatic methods and expressed in milligrams per deciliter. Total serum lipids were calculated using the modified Phillips formula: total serum lipids $=(2.27+$ total cholesterol $)+$ triglycerides $+62.3 \mathrm{mg} /$ $\mathrm{dl}$, assuming free cholesterol was $27 \%$ of the total $[13,14]$.

\section{Statistical Analysis}

Descriptive analysis included assessing data completeness, inspecting lipid distributions for informative values or outliers, and a comparison of the study population by pregnancy outcome to help inform model specification. Significance was assessed using Fischer's exact test or ANOVA depending on the type of variable. Total serum lipids and the individual lipid components were quantified by the timing of the sample and stratified by pregnancy outcome (i.e. birth, loss or no observed pregnancy). In the inferential stage of our analysis, we first used the fetus-at-risk model [15] to assess the association between the estimated rate of change in individual or total serum lipid measurements and timing of birth as measured by estimated postconception gestational age (in weeks). This model compares remaining fetuses at a given gestational age as the comparison group rather than live births. In addition, the fetus-at-risk model treats gestational age as a survival time instead of arbitrary definitions for pregnancy loss or preterm delivery. Specifically, we modeled the incidence of birth using a Cox proportional hazards model [16], where each birth or loss is compared with all fetuses alive at that specific gestational age. This approach is in keeping with the survival nature of pregnancy, and avoids erroneous comparisons of losses with births irrespective of the gestational age for each. Regression coefficients were used to estimate hazard ratios (HR), where an HR $>1$ indicates an increased risk of shorter gestation (pregnancy loss or preterm birth), whereas an $\mathrm{HR}<1$ indicates a decreased risk of shorter gestation (i.e. longer gestation and decreased risk of pregnancy loss or preterm birth). 
Table 1. Description of study cohort by observed outcome during follow-up

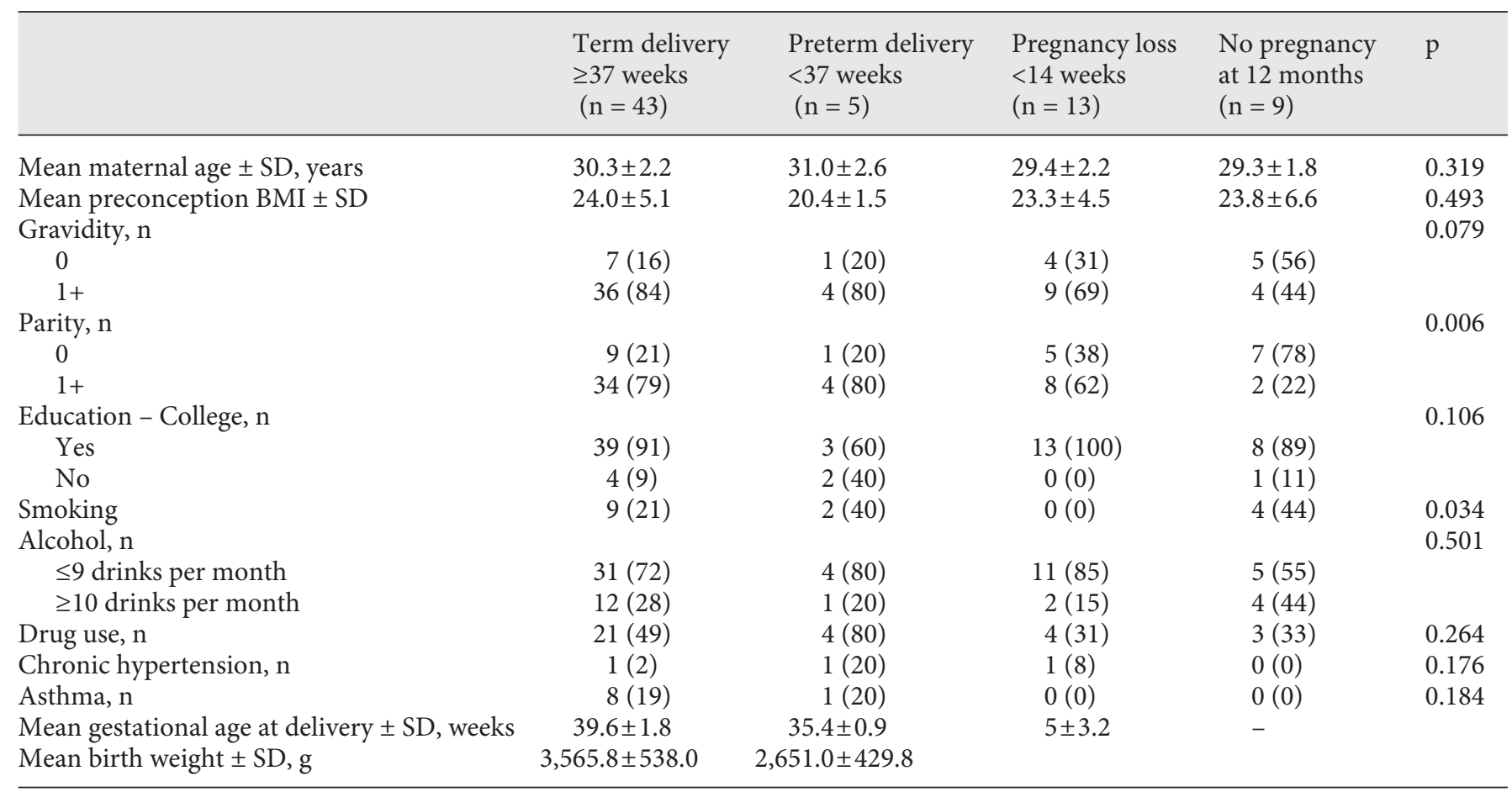

Values in parentheses are percentages. Statistical significance by Fisher's exact test for categorical and by ANOVA for continuous variables.

To quantify the change in either an individual lipid component or total serum lipids as a function of a woman's baseline, we estimated the daily rate of change in lipids by the following formula:

Pregnancy lipid measurement $(\mathrm{mg} / \mathrm{dl})-$ preconception lipid measurement $(\mathrm{mg} / \mathrm{dl})$

\section{Days of pregnancy}

Lipids were evaluated in both their continuous (milligrams per deciliter) and categorical (above and below the median) form. The preconception lipid concentration and daily rate of change were modeled separately. We ultimately decided to use daily rate of lipid change above and below the median, with above the median as the referent group in the final models. Other covariates in the model included: maternal prepregnancy body mass index (BMI) and cigarette smoking while attempting pregnancy (yes or no). Crude and adjusted HR (HR and AHR) are accompanied by $95 \%$ CI for assessing significance.

Traditional multinomial regression models also were performed to investigate the association between daily rate of changes in lipids (total serum and individual lipid components) with preterm ( $<37$ weeks) birth or pregnancy loss ( $<14$ weeks of gestation) compared with birth at term ( $\geq 37$ weeks), adjusting for the same confounders. Crude and adjusted odds ratios (OR and AOR) were calculated. We assessed the linear and nonlinear (i.e. U-shaped) associations between preconception total and individual lipids and pregnancy outcomes. Lastly, sensitivity analyses were performed that corrected for the timing (gestational age) of blood collection during pregnancy, given the observed negative daily rate of change in lipids in early pregnancy that became positive at approximately 8-10 weeks gestation. Instead of using the actual observed rate of change in the models, we used the woman's estimated rate of change at 8 weeks gestation.

For any missing individual lipid measurements $(\mathrm{n}=31)$, an Expectation-Maximization multiple imputation algorithm was used [17]. Statistical analyses were performed using SAS version 9.2 (SAS Institute Inc., Cary, N.C., USA).

\section{Results}

Sixty-one women (87\%) became pregnant, of which 43 women delivered at term ( $\geq 37$ weeks gestation), 5 women delivered preterm ( $<37$ weeks gestation), and 10 women experienced losses ( $<14$ weeks of gestation). Nine women (13\%) did not become pregnant. The mean interval, accompanied by its standard deviation (SD), between the preconception and calculated last menstrual period was 13.8 (SD: \pm 17.3 ) weeks, reflecting the varying time women required for becoming pregnant. The average gestational age at lipid sampling during pregnancy was 6.6 (SD: \pm 2.8 ) 
Table 2. Preconception and mean change in serum lipid concentrations by pregnancy outcome according to time of sampling

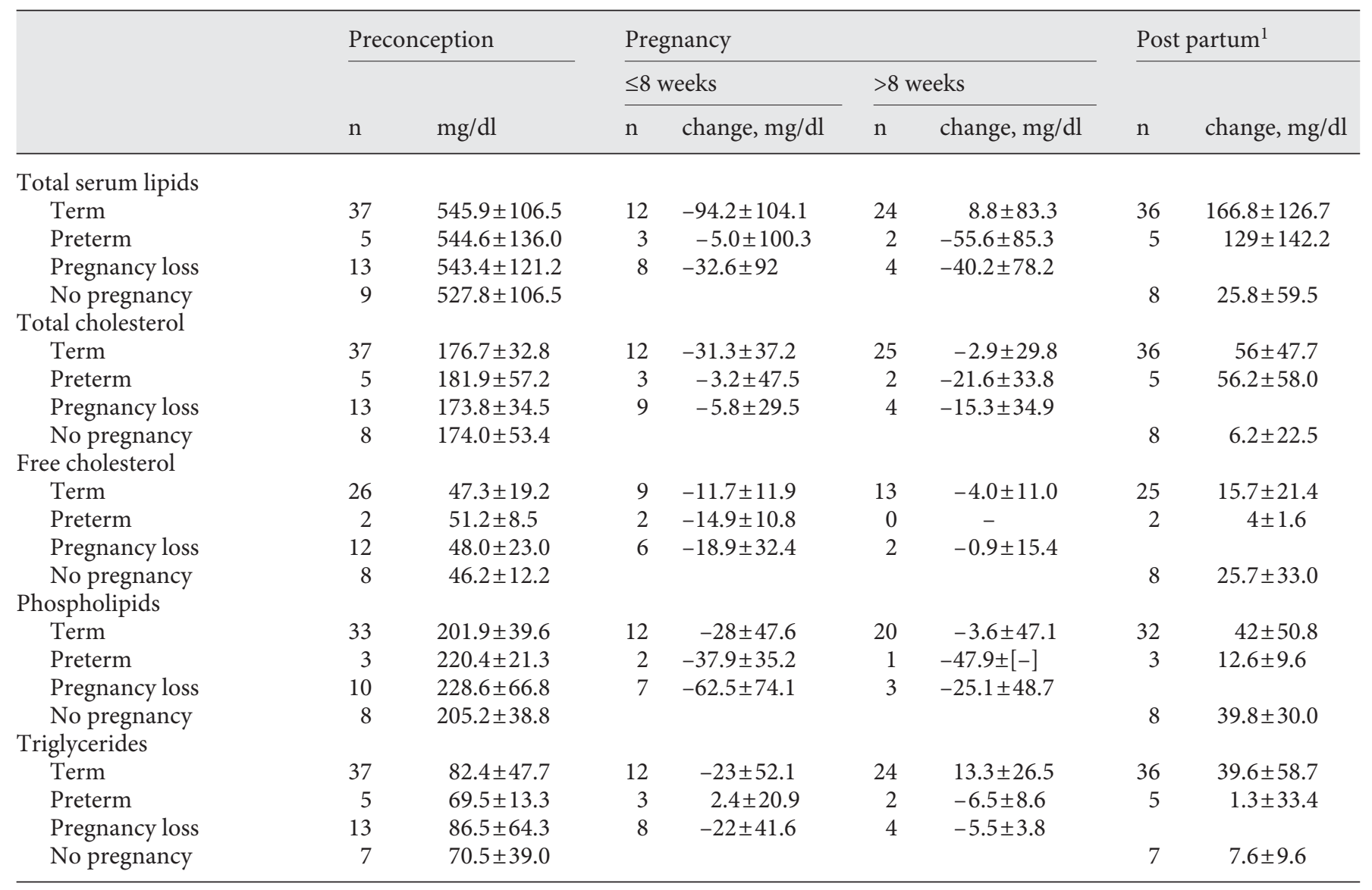

Values denote means \pm SD unless specified otherwise. Prenatal change refers to the difference between prenatal lipid concentration and preconception lipid concentration. For example, the mean change for total serum lipids at $\leq 8$ weeks included only the women with a prenatal sample $\leq 8$ weeks compared with the preconception sample in those women. Postnatal change refers to the difference between postnatal and prenatal lipid concentration. The following are significantly different between preconception and $\leq 8$ weeks ( $\mathrm{p}<0.05$ for two-sample t test): total serum lipids (term), total cholesterol (term) and free cholesterol (term).

${ }^{1}$ If no pregnancy, then sampled after 12 months. weeks for women who delivered at term, 5.5 (SD: \pm 1.0 ) weeks for women who delivered preterm, and 6.4 (SD: $\pm 2.4)$ weeks for women with a pregnancy loss ( $p=0.717)$, and post partum was 4.1 (SD: \pm 1.7 ) weeks after delivery.

The study cohort comprised mostly white married gravid women with a college education (table 1). Women who had a pregnancy were more likely to be multiparous (79\% for term and $80 \%$ preterm) compared with women experiencing a pregnancy loss (62\%) or without an observed pregnancy $(22 \% ; p=0.006)$. In addition, women with a delivery at term were less likely to be smokers (21\%) compared with women with a preterm birth (40\%) or without an observed pregnancy (44\%), while women with a pregnancy loss were nonsmokers $(\mathrm{p}=0.034)$.
Preconception means and mean changes in total serum lipids and individual lipid components are presented by timing of blood collection and pregnancy outcome in table 2 . Women with a preterm birth had a $5-8 \mathrm{mg} / \mathrm{dl}$ higher preconception total cholesterol and 3-4 mg/dl higher free cholesterol compared with preconception total and free cholesterol for women with other pregnancy outcomes, although these differences were not statistically significant. Preconception triglycerides were highest in women with a pregnancy loss and lowest in women with a preterm birth. The mean change in serum lipids from preconception to early pregnancy $\leq 8$ weeks of gestation was decreased. The mean lipid change between preconception and subsequent measurement in pregnancy, or 12 months later if no preg- 
Table 3. Daily rate of change in serum lipids (median) and the hazards of earlier delivery or pregnancy loss

\begin{tabular}{|c|c|c|}
\hline $\begin{array}{l}\text { Daily rate of changes } \\
\text { in serum lipids, } \\
\mathrm{mg} / \mathrm{dl} \text { per day }\end{array}$ & Unadjusted HR & $\mathrm{AHR}^{1}$ \\
\hline \multicolumn{3}{|l|}{ Total serum lipids } \\
\hline$-0.49,2.52$ & reference & reference \\
\hline$-6.43,-0.48$ & $1.02(0.56,1.86)$ & $0.99(0.55,1.79)$ \\
\hline \multicolumn{3}{|l|}{ Total cholesterol } \\
\hline$-0.12,0.92$ & reference & reference \\
\hline$-2.63,-0.11$ & $1.04(0.56,1.90)$ & $1.02(0.57,1.84)$ \\
\hline \multicolumn{3}{|l|}{ Free cholesterol } \\
\hline$-0.17,0.37$ & reference & reference \\
\hline$-2.07,-0.16$ & $0.99(0.54,1.79)$ & $1.10(0.60,2.03)$ \\
\hline \multicolumn{3}{|l|}{ Phospholipids } \\
\hline$-0.26,1.19$ & reference & reference \\
\hline$-5.22,-0.25$ & $1.12(0.61,2.04)$ & $1.13(0.62,2.07)$ \\
\hline \multicolumn{3}{|l|}{ Triglycerides } \\
\hline $0.00,0.99$ & reference & reference \\
\hline$-3.07,-0.01$ & $1.48(0.81,2.70)$ & $1.77(0.94,3.33)$ \\
\hline \multicolumn{3}{|c|}{$\begin{array}{l}\text { Values in parentheses denote } 95 \% \text { CI. Daily rate of lipid change } \\
\text { is calculated as (pregnancy measurement - preconception mea- } \\
\text { surement)/days of pregnancy. } \\
{ }^{1} \text { Adjusted for maternal BMI and cigarette smoking (yes/no). }\end{array}$} \\
\hline
\end{tabular}

nancy, is presented by lipid component and pregnancy outcome in online figure 1 (for all online suppl. material, see www.karger.com/doi/10.1159/000355100). Preconception triglycerides were highest in women with a pregnancy loss, but the mean decrease in pregnancy was marked compared with women who had a delivery at term or preterm. Preconception triglycerides were lowest in women with a preterm birth, and the mean change during pregnancy was flat compared with an increase in triglycerides observed in women who delivered at term. Preconception total cholesterol was highest in women with a preterm birth, with a mean change similar to pregnancies delivering at term. On average, women not achieving pregnancy experienced an increase in total lipids compared with women who achieved pregnancy.

Using regression techniques, preconception total and individual lipid components were not associated with length of gestation, or with risk of pregnancy loss or preterm birth. Women whose triglyceride concentrations were consistent with a negative daily rate of change from preconception to the prenatal measurement below the median ( -3.07 to $-0.01 \mathrm{mg} / \mathrm{dl}$ per day) had a nonsignificant 1.8-fold increased risk of pregnancy loss or earlier birth (AHR: 1.77; 95\% CI: 0.94-3.33; table 3). In the tra- ditional models, the rate of change for triglycerides below the median versus above the median was associated with an increased risk for pregnancy loss compared with term birth (AOR: 9.02; 95\% CI: 1.62-50.30; table 4). There were no other associations between individual lipid components and pregnancy outcomes when evaluating by the median (tables 3, 4).

More of the women who delivered at term were sampled at a later gestational age, while women who had a pregnancy loss were sampled earlier. Given the observed negative daily rate of change in lipids in early pregnancy that became positive at approximately $8-10$ weeks of gestation, sensitivity analyses were performed that corrected for the timing (gestational age) of blood collection during pregnancy. The relation between triglycerides and length of gestation was in the same direction, but no longer significant (AHR: 1.29; 95\% CI: 0.71-2.35). Similarly, the odds of pregnancy loss when comparing changes in serum triglycerides below the median rate of change with those above remained elevated, but the CI included 1 (AOR: 3.75; 95\% CI: 0.83-17.01). Regression coefficients for the rate of change in relation to gestational age as stratified by pregnancy outcome did not reveal any differences for triglycerides and most of the other measures.

\section{Discussion}

In women with early sampling, serum lipids initially decreased on average during early pregnancy compared with women with later sampling, where serum lipids started to increase on average above preconception levels around 10 weeks of gestation, except for triglycerides, which started to rise earlier around 7-8 weeks of gestation, with disproportionate rates depending on pregnancy outcome. The rate of change in triglycerides from preconception to early in pregnancy was different for women with a shorter length of gestation compared with women who delivered at term. Specifically, compared with an increase in the rate of triglyceride change observed in women who delivered at term, there was a decrease in the rate of triglyceride change in women with a pregnancy loss, and no change in women with a preterm birth. Though the numbers were small, preconception mean triglyceride levels were higher in women with a pregnancy loss at $<14$ weeks of gestation, and lower in women with a preterm birth at $<37$ weeks of gestation, compared with women with a term birth at $\geq 37$ weeks of gestation, and preconception total and free cholesterol were slightly higher in women with a preterm birth compared with other preg-
10

Gynecol Obstet Invest 2014;77:6-13 DOI: $10.1159 / 000355100$
Laughon/McLain/Sundaram/Catov/ Buck Louis 
Table 4. Daily rate of change in serum lipids (median) and pregnancy outcomes

\begin{tabular}{|c|c|c|c|c|}
\hline \multirow{2}{*}{$\begin{array}{l}\text { Daily rate of changes } \\
\text { in serum lipids, } \\
\mathrm{mg} / \mathrm{dl} \text { per day }\end{array}$} & \multicolumn{2}{|l|}{ Pregnancy loss } & \multicolumn{2}{|l|}{ Preterm delivery } \\
\hline & unadjusted OR & $\mathrm{AOR}^{1}$ & unadjusted OR & $\mathrm{AOR}^{1}$ \\
\hline \multicolumn{5}{|l|}{ Total serum lipids } \\
\hline$-0.49,2.52$ & reference & reference & reference & reference \\
\hline$-6.43,-0.48$ & $1.58(0.43,5.80)$ & $1.66(0.43,6.46)$ & $0.67(0.10,4.45)$ & $0.75(0.10,5.43)$ \\
\hline \multicolumn{5}{|l|}{ Total cholesterol } \\
\hline$-0.12,0.92$ & reference & reference & reference & reference \\
\hline$-2.63,-0.11$ & $1.91(0.53,6.91)$ & $2.04(0.54,7.67)$ & $0.79(0.12,5.31)$ & $0.86(0.12,6.25)$ \\
\hline \multicolumn{5}{|l|}{ Free cholesterol } \\
\hline$-0.17,0.37$ & reference & reference & reference & reference \\
\hline$-2.07,-0.16$ & $2.66(0.65,10.89)$ & $3.25(0.72,4.71)$ & $2.01(0.20,20.00)$ & $2.00(0.18,21.96)$ \\
\hline \multicolumn{5}{|l|}{ Phospholipids } \\
\hline$-0.26,1.19$ & reference & reference & reference & reference \\
\hline$-5.22,-0.25$ & $3.91(0.82,18.65)$ & $4.62(0.90,3.75)$ & $1.54(0.16,15.13)$ & $2.11(0.20,22.37)$ \\
\hline \multicolumn{5}{|l|}{ Triglycerides } \\
\hline $0.00,0.99$ & reference & reference & reference & reference \\
\hline$-3.07,-0.01$ & $7.74(1.49,40.26)$ & $9.02(1.62,0.30)$ & $0.98(0.15,6.54)$ & $1.30(0.17,9.82)$ \\
\hline
\end{tabular}

Values in parentheses denote $95 \%$ CI. Daily rate of lipid change is calculated as (pregnancy measurement preconception measurement)/days of pregnancy.

${ }^{1}$ Adjusted for maternal BMI and cigarette smoking (yes/no).

nancy outcomes. These findings highlight the fact that it likely is important to take into consideration the trajectory of change in relation to preconception concentrations when investigating the relation between maternal serum lipids and pregnancy outcomes.

Interpreting our findings in the context of the past literature is not straightforward, as our research is the first to utilize a prospective cohort design with the added strength of preconception recruitment, allowing for a preconception lipid profile. Still, our findings of a different rate of change in triglycerides being associated with an increased risk of pregnancy loss (decrease) or preterm birth (no change) are consistent with what has previously been reported, where a flatter rise in triglycerides was reported between early and midgestation in women with a preterm birth prior to 34 weeks of gestation [8]. Shorter gestation was primarily explained by pregnancy loss in our study, but the small cohort size restricted our ability to assess the association with preterm birth, since only 5 women delivered prior to 37 weeks gestation.

Prior studies without information on preconception lipids have also found associations between both lower and higher cholesterol in the first half of gestation and preterm birth, but it is unknown whether these findings were due to differences in preconception or changes dur-

Lipids and Length of Gestation ing pregnancy $[8,9]$. Our finding that preconception total and free cholesterol were, on average, higher in women who delivered preterm is consistent with a preconception cohort study by Catov et al. [2] where cholesterol in the highest quartile (>195 mg/dl) was associated with a 3.8fold increased risk of preterm birth prior to 34 weeks of gestation (95\% CI: 1.07-7.57). That study also found an increased risk of preterm birth between 34 and 37 weeks with preconception cholesterol in the lowest quartile (OR: 1.86; 95\% CI: 1.10-3.15). Our study did not find this relation, and there was no statistically significant relation between any preconception lipids and preterm birth. These discrepant findings might be explained by a lack of power in our study, or perhaps may indicate that it is not important so much where a woman starts but how lipid components change with pregnancy. The increase in lipids 12 months apart in women with no observable pregnancy was unexpected and warrants further investigation.

The only other preconception study by Magnussen et al. [18] in 2011 found that a more unfavorable cardiovascular profile, including high triglycerides, cholesterol, glucose and low high-density lipoprotein cholesterol, was associated with preterm birth and a shorter length of gestation. Perhaps such women have a poor adaptation to 
pregnancy manifested by a slower rise in lipids, in particular triglycerides. This interpretation is supported by the fact that atherogenic lipids are associated with inflammation, and excessive inflammation in the first trimester marked by an increased plasma C-reactive protein of $\geq 8$ $\mathrm{mg} / \mathrm{l}$ was shown to be associated with a 2.6-fold increased risk of preterm delivery [19]. The reason that the signal is for triglycerides and not for other lipid components is unclear, but it may be explained by the differences in rates of change in the individual lipid components. Triglycerides increase approximately 3 -fold during pregnancy from nonpregnant controls, compared with only a 0.5 fold increase for cholesterol, and they also start to change earlier in pregnancy than other lipid components, so the rate of triglyceride change might be the easiest signal to detect [10]. A larger cohort with a longitudinal analysis of fasting lipids from preconception to postpartum phases is needed to further explore this hypothesis.

Our study is limited by the fact that women who had a pregnancy loss were sampled after a loss was recognized in all but one woman. If a different timing of attainment explained all of our findings, one would expect that more lipid components would have an abnormal rise, not just one lipid component (triglycerides). The variation in timing of pregnancy sampling can also be considered informative to have ensured adequate representation of different gestational ages, since the critical time points for sampling lipids were unknown. Sensitivity analyses to correct for gestational age of sampling were reassuring, since the findings were in the same direction. Our findings are further corroborated by those from prior studies without preconception lipids that observed a flat rise in triglycerides in the first half of pregnancy being associated with a shorter length of gestation [8]. Lipid values were nonfasting, since samples were originally collected for biochemical analyses. However, it is unlikely that the differences between pregnancy outcomes could be explained by different eating patterns, and past research has also relied upon nonfasting lipids [18]. Further, women with a pregnancy loss may have less nausea and vomiting, allowing for higher food intake and, possibly, serum lipids. Nonfasting lipids may introduce error or precision of the estimates but not bias.

The major strength of our study was the ability to study the rate of lipid changes in relation to preconception concentrations. While the number of women was small in our study, it is comparable with the original comprehensive study of longitudinal assessment of lipids in pregnancy $(n=43)$ where gestational age at serum lipid attainment also varied between women [10]. Selection bias remains a consideration. However, there was no empirical evidence to support the assumption that lipid profiles varied by selection into the study or retention. Women were not aware of their serum lipid levels. Women who stated that they had not completed their families were recruited from the overall cohort. As a result, the eligible number of women was small (244 out of 30,000 questionnaires mailed in the original study, or $0.8 \%)$. Still, this finding along with the $39 \%$ reached for screening ( 1,031 out of 2,637 women in the original study who had indicated possible interest in becoming pregnant) was consistent with that estimated for the population level. Specifically, $<1 \%$ of women/couples of reproductive age were planning to become pregnant at any point in time, and $42 \%$ of eligible couples were successfully screened in another recent preconception study [20]. While prospective pregnancy studies are challenging, our results demonstrate they are feasible and provide a key window to pregnancy adaptation that is critical to healthy gestation. Our findings are consistent with earlier findings that a slow rate of change for triglycerides may be an early signal of pregnancy loss or earlier delivery.

In a prospective cohort of women planning a pregnancy within the following 6 months with longitudinal sampling, a slow rate of triglyceride change was associated with a shorter gestation. Women with a pregnancy loss or preterm birth had higher preconception triglycerides and cholesterol, respectively, which is consistent with a more atherogenic lipid profile. Our preliminary findings are exciting and raise the question of whether improving the lipid profile prior to achieving pregnancy improves pregnancy success. Given the current obesity epidemic with more women likely to have an atherogenic lipid profile prior to pregnancy, purposeful investigation may determine a relevancy of serum lipids in predicting adverse pregnancy outcomes. Serum lipids may offer promise for identifying pregnancies at risk for adverse outcomes, and hold potential for future targets of intervention.

\section{Acknowledgments}

This research was supported in part with funding from the Great Lakes Protection Fund (RM791-3021), the Agency for Toxic Substances and Disease Registry (H751ATH298338) and the Intramural Research Program of the Eunice Kennedy Shriver National Institute of Child Health and Human Development. DOI: $10.1159 / 000355100$
Laughon/McLain/Sundaram/Catov/ Buck Louis 


\section{References}

$>1$ Herrera E: Lipid metabolism in pregnancy and its consequences in the fetus and newborn. Endocrine 2002;19:43-55.

$\checkmark 2$ Catov JM, Ness RB, Wellons MF, Jacobs DR, Roberts JM, Gunderson EP: Prepregnancy lipids related to preterm birth risk: the coronary artery risk development in young adults study. J Clin Endocrinol Metab 2010;95: 3711-3718.

-3 Grundy SM, Cleeman JI, Daniels SR, Donato KA, Eckel RH, Franklin BA, Gordon DJ, Krauss RM, Savage PJ, Smith SC Jr, Spertus JA, Costa F: Diagnosis and management of the metabolic syndrome: an American Heart Association/National Heart, Lung, and Blood Institute scientific statement. Circulation 2005; 112:2735-2752.

4 Romero R, Espinoza J, Goncalves LF, Kusanovic JP, Friel L, Hassan S: The role of inflammation and infection in preterm birth. Semin Reprod Med 2007;25:21-39.

$>5$ Irgens HU, Reisaeter L, Irgens LM, Lie RT: Long term mortality of mothers and fathers after pre-eclampsia: population based cohort study. BMJ 2001;323:1213-1217.

$\checkmark 6$ Smith GC, Pell JP, Walsh D: Pregnancy complications and maternal risk of ischaemic heart disease: a retrospective cohort study of 129,290 births. Lancet 2001;357:2002-2006.

$>7$ Smith GD, Sterne J, Tynelius P, Lawlor DA, Rasmussen F: Birth weight of offspring and subsequent cardiovascular mortality of the parents. Epidemiology 2005;16:563-569.
-8 Catov JM, Bodnar LM, Kip KE, Hubel C, Ness RB, Harger G, Roberts JM: Early pregnancy lipid concentrations and spontaneous preterm birth. Am J Obstet Gynecol 2007;197: 610e1-e7.

9 Edison RJ, Berg K, Remaley A, Kelley R, Rotimi C, Stevenson RE, Muenke M: Adverse birth outcome among mothers with low serum cholesterol. Pediatrics 2007;120:723733.

10 Potter JM, Nestel PJ: The hyperlipidemia of pregnancy in normal and complicated pregnancies. Am J Obstet Gynecol 1979;133:165170.

11 Vena JE, Buck GM, Kostyniak P, Mendola P, Fitzgerald E, Sever L, Freudenheim J, Greizerstein H, Zielezny M, McReynolds J, Olson J: The New York Angler Cohort Study: exposure characterization and reproductive and developmental health. Toxicol Ind Health 1996;12:327-334

12 Bloom MS, Buck Louis GM, Schisterman EF Liu A, Kostyniak PJ: Maternal serum polychlorinated biphenyl concentrations across critical windows of human development. Environ Health Perspect 2007;115:1320-1324.

13 Phillips DL, Pirkle JL, Burse VW, Bernert JT Jr, Henderson LO, Needham LL: Chlorinated hydrocarbon levels in human serum: effects of fasting and feeding. Arch Environ Contam Toxicol 1989;18:495-500.

14 Bernert JT, Turner WE, Patterson DG Jr, Needham LL: Calculation of serum 'total lipid' concentrations for the adjustment of persistent organohalogen toxicant measurements in human samples. Chemosphere 2007;68:824-831.
15 Joseph KS: Theory of obstetrics: an epidemiologic framework for justifying medically indicated early delivery. BMC Pregnancy Childbirth 2007;7:4

16 Cox DR: Regression models and life-tables. J R Stat Soc Series B Stat Methodol 1972;34: 187-220.

17 Horton NJ, Kleinman KP: Much ado about nothing: a comparison of missing data methods and software to fit incomplete data regression models. Am Stat 2007;61:79-90.

18 Magnussen EB, Vatten LJ, Myklestad K, Salvesen KA, Romundstad PR: Cardiovascular risk factors prior to conception and the length of pregnancy: population-based cohort study. Am J Obstet Gynecol 2011;204:526e1-e8.

19 Pitiphat W, Gillman MW, Joshipura KJ, Williams PL, Douglass CW, Rich-Edwards JW: Plasma C-reactive protein in early pregnancy and preterm delivery. Am J Epidemiol 2005; 162:1108-1113.

20 Buck Louis GM, Schisterman EF, Sweeney AM, Wilcosky TC, Gore-Langton RE, Lynch CD, Boyd Barr D, Schrader SM, Kim S, Chen Z, Sundaram R: Designing prospective cohort studies for assessing reproductive and developmental toxicity during sensitive windows of human reproduction and development: the LIFE Study. Paediatr Perinat Epidemiol 2011; 25:413-424. 